\title{
The Influence of Patriarchal Clan System on the Legal Status of Chinese Women in Marriage and Family-_Suggestions on Promoting the Implementation of Anti Domestic Violence Law
}

\author{
Siyu Feng \\ School of Si chuan University, Sichuan 610000, China. \\ bern1937@outlook.com
}

\begin{abstract}
Domestic violence is a social pain that affects family harmony and social stability. Through the comparison of the status of Chinese ancient and modern women in marriage and family, this paper shows that although the status of Chinese women's status is gradually rich and the perfect law can block their "fist", the unequal influence of the patriarchal ethics system and the feudal ideology still exists deeply. Especially in some economically backward areas and families with weak legal consciousness, vicious family rules are also preventing the society from realizing the equality of men and women in the true sense. Finally, this paper makes a brief analysis of how to deal with the problem of domestic violence and protect the legitimate rights and interests of women by revealing the problems of the anti-domestic violence law.
\end{abstract}

Keywords: Family violence law; Patriarchal clan system; Marriage and family; Improvement of antidomestic violence law.

\section{从家暴现象看宗法制对中国女性在婚姻家庭中法律地位的影响 一浅谈促进反家暴法有效实施的建议}

\author{
馮思宇 \\ 四川大学, 成都 中国
}

摘 要: 家暴这一社会隐痛, 影响着家庭和谐与社会稳定。本文通过对中国古代和现代女性在 婚姻家庭中地位的对比, 显示出中国女性的地位虽然逐渐富有弹性的空间以及完善中的法律 也能为她们阻挡家暴的 “拳头”，但宗法伦理制度和男尊女卑、封建传统思想造成的不平等 影响依然深刻存在。特别是部分经济落后的地区和法律意识淡薄的家庭, 恶性 “家规” 更是 阻碍社会实现真正意义上的男女平等。最后本文通过揭示出台的反家暴法存在的问题对如何 更为有效的处理家暴问题和保护妇女合法权益等作出简要分析。

关键词: 家暴法; 宗法制; 婚姻家庭; 反家暴法完善建议

\section{1. 前言}

家暴是一个普遍又长期存在的话题。家暴顾名思义就是家庭暴力，是指发生在家庭成员之间 的, 以殴打、捆绑、禁闭、残害或者其它手段对家庭成员从身体、精神、性等方面进行伤害 和摧残的行为。家庭暴力最直接作用于受害者的身体, 造成受害者身体疼痛、重伤甚至死亡, 不仅如此, 受害者的精神也会饱受痛苦。对长期遭受家庭暴力伤害的人来说, 她们往往选择 “以暴制暴” 的极端行为以发泄情绪, 这势必会给社会带来更多不稳定因素, 危害社会安定 的局面 [1]。

因为家庭暴力问题是一个长期以来在世界各地普遍存在的问题，所以从世界范围来看，上世 纪 60 年代起人们就开始系统地研究这一问题 [2]。1986 年, 联合国曾举办过一个关于 “家庭 暴力” 的专家委员会。专家委员会在报告中指出：即使是偶然发生家庭暴力事件，如不立即 采取紧急干预, 暴力行为往往就会一再重复发生并趋于严重。同时调查普遍显示: 在家暴事 
件中受害者极大部分是女性, 所以家暴也被社会认为是男性权力压迫女性的典型表现。世界 卫生组织 (WHO) 2013 年 6 月 20 日公布的一项调查显示, 目前全球约三分之一的女性曾遭受到 爱人或丈夫的暴力伤害, 其中亚洲以及中东地区以 $37.7 \%$ 的比例为最多。WHO 还表示从世界规 模的资料研究显示, 目前仍有 30\%的女性遭受虐待。关于家暴对女性的伤害, 临床数据显示: 受到家暴的女性患精神疾病以及不正常生育的几率大大高于正常女性, 正如世界银行认为, 暴力对女性来说与癌症一样是育龄妇女死亡和丧失生存能力的重要原因。

在我国, 全国妇联公布的中国妇女社会地位调查报告显示, 与十年前的调查相比, 妇女的健 康状况、女性受教育的程度、女性的经济能力等都有了明显的提高。不过我国仍有 $24.7 \%$ 的 女性在婚姻生活中遭遇到不同形式的家庭暴力 [3]。这让我们不免疑问, 在女性的受教育程度 和经济能力都大幅度提高的今天为何仍然有近乎四分之一的女性生活在家暴的阴影下。并且 众所周知, 家庭暴力引起的后果是多方面的, 它不仅极大地危害着妇女儿童的身心健康, 并 且是一个十分突出的社会问题。比如生活在发生家庭暴力的家庭中的孩子通过耳濡目染、潜 移默化，就大大增加了他们成长后使用暴力的可能性。

\section{2. 我国反家暴法出台后的理想与现实}

2015 年 12 月 27 日第十二届全国人民代表大会常务委员会第十八次会议通了《中华人民共和 国反家庭暴力法》，2016 年 3 月 1 日《中华人民共和国反家庭暴力法》正式實施。这对预防 和制止家庭暴力, 保护家庭成员的合法权益、维护平等、和睦家庭关系、促进社会稳定具有 重大意义 [4]。可是尽管有法律保护妇女不受虐待, 家庭暴力案件的发生率依然有上升的趋势。 根据中国妇女联合会权益部门统计, 在目前的家庭暴力事件中, 丈夫对妻子实施暴力的仍占 绝大多数, 家庭暴力的受害者 $90 \%$ - $95 \%$ 是女性。并且众多家庭在面临此问题时, 多数选择 “关上自家门，沉默”，认为家暴一般不能让外人知道，“家丑不可外扬” “疏不间亲”，更 有部分男性认为修理老婆天经地义。在中国传统法观念的影响下被害人其近亲属、邻居、同 事即使知道, 也不想报案, 这导致司法机关难以及时发现家暴犯罪事实, 发挥法律的作用。 同年 3 月，笔者针对家暴问题在成都市双流县文星镇进行小范围相关情况的样本调查。

1. 调查时间 2016 年 3 月 10 日到 2016 年 3 月 13 日。

2. 调查方法及调查对象

本次调查形式: 采取街采调查问卷为主;

年龄阶段：以中年妇女为主要对象;

调查问卷数量：共发放调查问卷 120 份，有效率 $94.1 \%$ 。

3. 调查问卷的结果

本次社会调查设计的调查问卷, 包含受调查者对家庭暴力的理解、施暴的原因、方式、遭受 时所采取的应对措施等各方面问题展开调查。

通过调查问卷的整理与数据统计, 数据显示如下:

根据此调查表的不完全结果表明, 近年来, 经济环境、法制建设不断改善, 妇女维权意识不 断提高, 但是家庭暴力现象并没有消除。在当今这个男女平等观念深入人心的时代, 被家暴 的女性中, 仍然多数人不愿意被周围人知道, 也不会选择报警。对遭受家暴的中国妇女来说, 影响声誉是她们所担心的普遍问题。为何中国女性会有如此大的思想负担? 究其根本, 主要 原因在于中国浓厚的道德伦理根源, 从思想道德源头给与了家暴滋生的土壤 [5]。 
表1《家暴问题调查表》

\begin{tabular}{|c|c|c|c|c|}
\hline $\begin{array}{c}\text { 题号 } \\
\text { 选项 }\end{array}$ & A & B & C & D \\
\hline 1. 是否遭遇过家暴 & 没有33\% & 有67\% & & \\
\hline 2. 起因 & 婚外情27\% & 子女教育矛盾23\% & 家庭收入低17\% & 其他33\% \\
\hline 3. 是否作伤情鉴定 & 是10\% & 不是56\% & 看情况而定 & \\
\hline 4. 是否愿意让周围人知道 & 愿意13\% & 不愿意51\% & 看情况而定 & \\
\hline 5. 是否报警 & 不会报警60\% & 情况严重报警24\% & 每次都报警3\% & 不确定13\% \\
\hline 6. 是否需要专门机构 & 没必要7\% & 可有可无32\% & 需要61\% & \\
\hline
\end{tabular}

\section{3. 宗法制家族意识特征与对我国伦理体系和 “国法” 变革的深刻影响}

宗法家族制在中国历史上由来已久, 确立于夏朝, 发展于商朝, 完备于周朝, 是用父亲血缘 关系的亲疏来维系等级、巩固国家统治的政治制度。它确立了父权在家庭中的统治地位，影 响于后来的各封建王朝。在中国古代, 家族组织占据重要位置: 在国家的稳定时期, 中央集 权用宗法家族制控制基层社会; 在战乱以后中央集权用宗法家族制粘合基层社会。经过千百 年的延续继承，宗法家族制度已然成为了中国社会架构的重要血液 [6]。致力于研究中国问题 的著名美国汉学家费正清在《中国与变革》中指出：“首先，是家庭而不是个人或教会组成 了中国最重要的单位。每个人的家庭是他经济资助、安全、教育、社会交往和娱乐的主要来 源，祭祀甚至是个人重要宗教活动的中心。在儒家的五种著名关系中：君臣、父子、夫妻、 兄弟、朋友, 其中有三种由亲属关系所决定的。中国整个伦理体系倾向于家庭为中心，而不 是以上帝或国家为中心。”

今天，中国已经没有宗族制度和封建家族组织，但家族观念和宗法思想仍广泛而深刻地植根 于中国人的头脑中并且影响着中国人的价值取向和行为方式。虽然, 我们不能否定宗法家族 制的积极作用，譬如注重提倡长幼次序、忠孝，使国人养成了谦和忍耐、温良恭敬的社会美 德, 但是男尊女卑的封建观念以及对人权和自由的压制依然是当今家庭和女性的毒性深渊, 影响着受害者对家庭暴力的一再容忍，直至麻木。在 2014 年央视 12 播出的《中国反家暴纪 事》节目就真实反映了很多被家暴的女性正是因为丈夫 “一家之主” 的夫权封建观念作崇而 过得生不如死。对于如何消弭这可怕的思想毒瘤，值得深思。

2011 年，中国疯狂英语创始人李阳的美国老婆金・李向媒体公开了自己被打得鼻青脸肿的照 片，她表示被家暴期间她多次向警方求助，但是都被警方以 “家务事不好参与” 和并无重大 伤害无法立案规劝回家。因为多次报警未果, 她只能向新闻媒体求助。可见在中国, 即使是 执法人员也存在着“宁拆十座庙, 不毁一桩婚” 的中国式传统思维。又如 “李红霞家暴事件”, 李红霞将自己被家暴一事告诉其母亲, 母亲劝她内部解决, 不要张扬出去。李红霞的母亲段 柳枝（音）说，她不愿意女儿离婚，因为离婚在县城里会坏了“名声” 的。

千百年的封建制社会里, 夫权思想渗透至社会的每一个角落。所谓夫权, 《法学大辞典》中 将其解释为: 夫妻关系中, 丈夫控制支配妻子的权利。在夫权统治下, 夫妻之间是一种尊卑, 主从关系, “妻以夫为天”。在我国, 对于家暴这一问题的公众关注相对推迟, 尽管新中国 成立以来，男女平等作为基本国策逐渐深入人心，但传统文化积淀仍影响着社会对家庭暴力 的态度。受夫权思想观念、家庭伦理的影响, 打老婆、打孩子等家庭暴力行为仍然被多数社 会人视为 “家务事”，外人不好干涉，民间更是有很多相关的俗语：“夫妻没有隔夜仇”、 
“床头打架床尾和” 等。许多家庭暴力正是打着这样的幌子肆意横行, 而旁人在目睹这种暴 力时，也多半是袖手旁观，不会将它与 “违法犯罪” 联系在一起 [7]。

3. 1. 家长制下古代女子的社会地位

在宗法制出现之前中国妇女的地位还是很高的, 譬如在夏朝开国时还是母系氏族, 商代也是典 型的母系氏族社会。然而随着周朝宗法制的出现, 制定了嫡庶、庙祭等一系列制度, 至此中 国妇女的社会地位就开始逐渐沦落。按照周礼的规定, 男性贵族可以娶妾多人, 这使得已婚 妇女地位卑下, 婚姻关系能否维系完全取决于丈夫的好恶。丈夫愿意维系, 她是丈夫的附庸; 丈夫不愿维系，就要被丈夫扫地出门。在这种婚姻制度下夫妇间只是主从关系，其等级贵贱 全由男性来确定。同时期的文学作品《诗经》中的一些诗词, 如《邦风・谷风》、《王风・中 谷有蓷》，均反映了当时弃妇的悲戚之声。

在后周春秋战国时期, 妇女社会地位更加沦落, 她们被男权主义者视为卑贱的性别而普遍遭 到歧视与贬损 [8]。例如, 孟子有一次突然走进卧室, 见妻子张开两腿如箕而坐, 便十分气愤 地告诉他母亲, 此 “妇无礼, 请去之” 反映出古时把妇女的身体看做肮脏和不纯洁的象征。 再如《战国策・赵策四》中记载, 某客规棟赵王, 说夫人和美人, 都能使国君昏醉, 以致国事 不可收拾 $[9]$ 。社会上层更有 “杀妻求将”、“以女殉葬” 等等的风俗。同时这种社会性别伦 理文化不仅盛行在达官贵族，老百姓之间也是如此，如下层民众 “卖妻妇以救赡父母” 就是 真实写照 [10]。于是女人身上的鬼力和光鲜都被男权主义赋予了卑鄙下流的字眼, 这极度不 平等的两性关系使得我国古代妇女在极限膨胀父权或夫权的社会下犹如蝼蚁一般卑微存活。 西汉武帝之后, 儒学思想逐渐成为中国官方的正统思想。但是儒学依然继承发挥了男尊女卑 的观念。例如唐太宗李世民宗室女一一文成公主入藏的故事。唐太宗李世民即位后, 国力日 益强大, 达到鼎盛, 但西域边关战火不断, 百姓生灵涂炭, 士兵血流成河, 和平呼声日益高 涨。西域诸国唯有吐蕃最为强大, 于是唐太宗将文成公主作为和亲休战的政治工具远嫁西藏。 在我们感慨这嫁入地域些乡的女子的和亲造就了唐与吐蕃二百多年没烽火、为吐蕃带去中原 灿烂文化的同时，却不曾着想她作为政治工具不能回归只能想念，饱受离乡的苦楚。

宋朝时期, 理学家极力主张 “存天理、灭人欲” , 在婚姻家庭制度方面, 荋视妇女的权益。

《宋史》记载中所谓的列女几乎全都是保持贞操、不事二夫的节妇 [11]。在当时 “贞洁顺女” 成为好女人的标准, 这其实是变相压制妇女的婚姻自由, 鼓吹男尊女卑的封建礼教 [12]。并 且宋朝法律规定, 妻告夫者, 即使所告为实, 也要 “徒二年”。这使得法律名存实亡, 妇女依 然是家庭的附属品。明清时代对于妇女的生活自由和婚姻自由的压制更是达到了前所未有的 残酷程度。明清时期由于统治者的腐朽, 统治苃苃可危。出于维护统治的需要统治者不断强 化对妇女守节的推崇和提倡, 麻木妇女的思想。与此同时封建的宗族势力也有了进一步的增 长, 尤其在广大的乡村, 迫害妇女、剥夺妇女再婚权利的宗族习惯法起着主要的调整功能。 因此, 妇女要想成功地再嫁, 首先就要遇到极其强大的宗族势力的阻碍, 于是深受封建礼教 “残害” 的殉夫妇女大量涌现。

3. 2宗法制---性别歧视思想的总根源

我国 1954 年就将男女平等写入了宪法。60 多年来, 在党和国家的高度重视和大力推进下, 男 女平等已经有了长足的发展。但遗憾的是, 至今仍看不到它的尽头。社会学者普遍认为造成男 女不平等的源头就在于宗法制度。宗法制度作为古代调整家族关系的制度，起源于父系氏族 社会对祖先的崇拜, 从继承和发展氏族社会父系家长制的过程中演变而来, 巩固了父权在家 庭中的统治地位 [13], 成为王族贵族按血缘关系分配国家权力, 建立世袭统治的重要依据。 在宗法家族血缘社会中, 婚姻被视为人伦之始, 婚姻成立的终极目的则是祭祀祖先与延续血 胤。父权家长制家庭普遍实行 “一夫多妻制” ，所以作为婚姻关系主体的男女双方个人的感 情和利益在这种婚姻观念中是微不足道的, 可以不予考虑。譬如宗法理论及宗法社会有 “出 妇”之说, 汉代统治者为确立家庭中丈夫的统治地位, 还制造了 “夫为妻纲” 的理论。《礼 记・郊特牲》言：“妇人，从人者也，幼从父兄，嫁从夫，夫死从子”，此 “三从”言论反映 出古代女性并没有家庭地位, 立命之本只能是依附男性。有的家族更是规定苛刻: 妇女要 “孝 
事公姑, 和处妯娌, 顺相丈夫, 女工习尚, 中馈勤白力, 慈剑贞洁” , “不幸寡居, 铁石, 白 首冰霜” [14]。所以，古代寡妇很多，立了不少所谓贞洁牌坊，但这不过是给 “男尊女卑” “压制人性”一个正当的头衔。相比之下，男人三妻四妾，却是十分正常的事。不仅如此， 在宗法制下家庭继承方面也突出地表现为 “父权统制, 男尊女卑” 的夫妻不平等现象: 血缘 集团在世系排列上完全排斥女性成员的地位，女性在继承方面完全没有权力。

两千多年来, 中国长期处在封建宗法制社会中。封建宗法制的社会结构和家庭结构, 都是以 男子为中心、为主体, 妇女处于无权的附庸地位。在婚姻家庭关系中, 男女结为夫妇, 女子 被娶到男方家庭中来, 主要是成为生育繁殖的机器, 生子育孙完成传宗接代的任务 [15]。 如今, 妇女的政治、经济地位和自主自立能力都已有很大提高, 但以 “男性传宗、女到男家” 为特征的宗法制思想依然浓厚, 重男轻女的现象依然存在。“立嫡以长不贤, 立子以贵不以 长”、“门当户对” 等古代婚姻家庭中的 “礼法” 原则在现今生活中依然深刻影响着中国人 的婚姻家庭观。若妇女的法律地位要做进一步的提高, 则必须充分认识到传统观念的消极影响, 以更切实有效的制度和思想建设来保障妇女的人权与自由, 完善和谐平等的婚姻家庭关系。

3. 3宗法制下的恶性 “家规”与 “国法”

俗话说, 国有国法, 家有家规。家与国同源是宗法社会最鲜明的结构特征, 并且在中国封建 社会被长期保留。家庭与国家在组织结构方面具有共同性, 也就是说不论国家或家庭, 他们 的组织系统和权力结构都是其成员均应无条件遵守的严格父权家长制。

在父系宗法制时代，家规常常凌驾于国法之上。“普天之下莫非王土，率土之滨莫非王臣” 就是天下第一大家规, 皇帝的家规自然大过国法。所以虽然由中国历朝历代国家制定的法律 规范一一国法, 是外部控制系统, 但其实质不过是以国家正式制度的形式出现的维护父权家 长制的宗法伦理。所以说当时的国法与家规不过都是由社会的原始习俗嬗变而来的; 法律制 度中对亲属或家庭成员间暴力行为的处理, 都是以维护伦理纲常和家长制度为基本原则的。 归根究底 “国法”依然没有维护妇女不受家庭的暴力 [16]。

两千多年来中国在传统宗法伦理的基本理念的指导下、宗法礼教为基本内容的影响下, 家庭 成员之间的暴力行为由家庭内部处理的封建思想并没有随历史发展而退去反而影响深远, 难 以更改。据 2016 年 4 月时代报报道: 浙江台州一名女子李云（化名）结婚 8 年以来长期遭到 丈夫家暴虐待，去年 4 月丈夫将其鼻子割掉。此家暴案例的背后，突显施暴者法律意识的淡 薄, 作为丈夫漠视了妻子的人权和合法权益, 并没有认识到自己的行为已经触犯法律。从今 年 3 月 1 日开始, 我国反家暴法正式实施, 但 “徒法不足以自行” , 反家暴法的出台只是从 根本上解决家庭暴力问题的一个新开端, 反家暴法要真正落地, 尚有很长的路要走。这些规 定只有最终落实到家庭生活的每一个环节、遏制住家庭内部的暴戻之气, 家暴悲剧才会最大 限度地得到避免。

\section{4. 反家暴法存在的问题}

4. 1. 未明确赋予警察对施暴者强制逮捕的权利 2009 年, 年仅 26 岁的北京女孩董珊珊被其丈夫殴打致死, 期间八次报警 (其中四次是自己报 警），都被警方以 “不好管，现在还是夫妻。”等为由拒绝了她及她家人的报警。2016 年反 家暴法实施以后, 董珊珊家暴事件再次获得关注, 有一部分原因是出台的《中国反家庭暴力 法》依然没有规定警察的强制逮捕权利，这使得人身保护令难以奏效。

中国警察的执行力弱常常被人诟病。在中国传统思想的影响下, 中国警察对家庭暴力多本着 “宁拆十座庙, 不拆一桩婚” 的原则进行劝解, 除非受害人向法院提请诉讼, 否则警察无权 逮捕加害者。在董珊珊事件中董珊珊与丈夫王光宇于 2008 年下半年结婚, 2009 年 3 月, 她 第一次向家人和警察披露婚后经常遭到王的殴打。从这时到她死亡, 短短几个月中间, 她及 家人曾先后八次向警方报告王的暴力行为, 曾提起过离婚诉讼, 但所有这些努力都未能挽救 她的生命。不得不承认的是即使反家暴法的出台也无法立刻彻底改变中国警察对于处理家暴 的执行力度。 
相反美国宪法第十四条修正案规定：“任何州不得在未经正当程序的情况下，对任何在其管 辖下的人, 拒绝给予平等的法律保护。” 同时, 美国法律赋予警方强制性逮捕施暴者的权力:

“警方在其有正当理由认定某人显而易见实施家庭暴力时, 即使不是现行犯, 亦得逮捕即无 令状逮捕。”[17]也就是说如果警察部门基于性别或其他原因拒绝逮捕施暴人，那么受害女 性就可以依法对警察部门提出控诉。

4. 2 反家暴法的惩罚力度较弱

反家庭暴力法第 5 章第 33 条规定： “加害人实施家庭暴力, 构成违反治安管理行为的, 依法 给予治安管理处罚; 构成犯罪的, 依法追究刑事责任。”那么据此理解：目前家暴最严重的 惩罚, 是按中国《刑法》规定的以虐待罪判处。《刑法》在第 260 条规定了虐待罪, 该条第 1 款规定：“虐待家庭成员，情节恶劣的，处两年以下有期徒刑、拘役或者管制。”第 2 款规 定：“犯前款罪，致使被害人重伤、死亡的，处两年以上七年以下有期徒刑。”也就是说虐待 最高刑期为 7 年。其惩罚力度远远低于故意伤害，致人死亡罪或者其他可判处 10 年以上有期 徒刑、无期徒刑甚至死刑的罪名。

\section{5. 总结与建议}

5.1 反家暴法的有效实施需社会文化的支持

任何法律从出台实施到效应的最大化都是一个长久且涉及广泛的过程, 因为任何新制定的法 律都不可能是尽善尽美的, 都会存在一定的缺憾, 所以法律的有效实施还需要靠思想建设、 社会文化来支持弥补。反家暴法也不例外，特别是在一个被 “三纲五常”、“三从四德”、

“家丑不可外扬”等传统观念深刻影响的国家。

自古中国人好面子, 遭遇家庭暴力不少人都选择哑忍, 官府也是不告不究。所以要想让家暴 悲剧减少，要从思想上文化上对女性进一步的解放，特别是还要对部分经济落后的地区和法 律意识淡薄的农村家庭妇女进行反家暴法律意识的唤醒 [18]。反家暴法第 2 章第 6 条规定:

“国家开展家庭美德宣传教育, 普及反家庭暴力知识, 增强公民反家庭暴力意识。工会、共 产主义青年团、妇女联合会、残疾人联合会应当在各自工作范围内, 组织开展家庭美德和反 家庭暴力宣传教育。广播、电视、报刊、网络等应当开展家庭美德和反家庭暴力宣传。学校、 幼儿园应当开展家庭美德和反家庭暴力教育。” 俗话说 “立法更要普法, 治暴更要治心”, 变治标为治本。反家庭暴力法出台后学校、幼儿园、医疗机构、居民委员会、村民委员会、 社会工作服务机构、救助管理机构等社会主体也要及时履行发挥相应的义务和责任, 让更多 的人认识到家暴已经不是家庭私事, 在全社会形成惩恶扬善的正确导向, 唤起弱势群体的自 我保护意识。

\section{2. 设立专业处理家暴的机构}

家庭暴力产生的真正根源在于男女不平等以及社会观念中歧视女性的传统思想。所以，要消 除家庭暴力, 必须努力创造一个男女真正平等的社会环境 [19], 并且需要出现更为专业和有 效地机构, 为受害人提供及时且专业的帮助和支持。

从现实中, 参考《家暴问题调查表》（表一）的数据可以看出：61\%的女性迫切希望国家建立 专业的处理家暴的机构。虽然法律规定妇联、居民委员会、村民委员会以及当事人所在单位 都有预防、制止和调解家庭暴力纠纷的责任。但是正如在董珊珊案件里我们可以看到虽然中 国的法律明确规定相关部门和组织都有预防、制止家庭暴力的责任，但事实是这些部门对防 治家庭暴力责任的认识和承担却是如此虚无，以至于并没有任何一个部门起到实质性作用。 在董珊珊案中, 最令人痛心的不是施暴者的凶残, 而是系统性的怠慢和姑息, 更令人心寒的 是他们的作为看起来却并无违规之处。

目前, 我国法律援助的对象主要是刑事审判中因经济困难而不能聘请律师的被告人。对于家 暴中的受害者是没有专业的法律援助机构的，相关部门之间又像踢皮球一样推卸责任，避免 问责，造成受害妇女求助无门的宕境。所以笔者建议反家暴法出台后，执行机制上应当做进 
一步细化, 最好能够成立专业的处理家暴的机构, 切实保障家庭成员特别是妇女儿童权益, 把家暴的悲剧降到最低。

\section{References}

[1]. Lin Xiuxiong, "The study of marriage and family law". China University of Political Science and Law Press, 2000

[2]. Rong Weiyi, Song Meiya, "opposed domestic violence against women". China Social Science Press, 2002,303-305.

[3]. Liu Yamei, "Women's studies". China National Women's Federation Press ,2003 (1), 66-72.

[4]. Li Derong, "Social perspective and Legal Reflection on domestic violence". 1995 World Conference on women 5th Anniversary Symposium.

[5]. Zhang Boying, "The contemporary Chinese citizen's Moral Construction -- the perspective of national ethics and civil society ethics" [M], Tianjin Academy of Social Sciences Press , 2001.

[6]. Zhao Gang, "The review of law". School of law, Wuhan University Press, 1998 (2), P 87-93.

[7]. Lan Xiang Dong, "Discussion on several issues related to domestic violence". Reprint by "democracy and the legal system", 2002.

[8]. Liu Zhongshu, "Journal of Social Sciences .Jilin University, China,1987 (4), P 36-39.

[9]. Zhan Guo CE Volume fourteen "Chu CE Yi Jiang Yi Jun Yu Ling Jun" [M] Liu Xiang, Shanghai ancient book publishing house Press, 1985.

[10]. Qiu Guozhen, Lai Shiqiu, "Folk culture and social status of women." Folklore Studies ,Press 2005 (2),P 41-49.

[11]. Huang Zhengyuan, "Love for the sea", Solidarity Press, 2015, P 21.

[12]. Wang Jinling, "Local research and experience in Feminist Sociology." Shanghai People Press, 2002.

[13]. Zhang Guohua, "New edition of Chinese legal thought". Beijing University ,China, 1998.

[14]. Dong Jiazun, "Research on marriage history in ancient China". Guangdong people Press , 2010 .

[15]. Cao Shi Quan, "Marriage and family law". Peking University,China, 2004,(08),p 184-188.

[16]. Deng Zhenglai, "patriarchal clan structure in the ancient civil dispute settlement mechanism", Law Press, 2000.

[17]. A review of contemporary American feminism(1999)". Information on: http://www, teachercm com/Zxyw/Zwsb/2006-9/7/ 20060108165750994.

[18]. Wang Xiaodan, "Investigation and Reflection on rural domestic violence in border areas", Southwest Nationalities College Press, 2002 (10).

[19]. "Anti domestic violence working group" Reporting "China: anti-domestic violence in action", 2000 